\title{
Towards clinic-friendly solutions for patient trials in breast cancer phase contrast imaging
}

Sarah J. Lewis, Timur E. Gureyev, Patrycja Baran, Seyedamir Tavakoli Taba, Serena Pacile, et al.

Sarah J. Lewis, Timur E. Gureyev, Patrycja Baran, Seyedamir Tavakoli Taba, Serena Pacile, Christian Dullin, Giuliana Tromba, Daniel Hausermann, Andrew Peele, Darren Lockie, Patrick C. Brennan, "Towards clinic-friendly solutions for patient trials in breast cancer phase contrast imaging," Proc. SPIE 10718, 14th International Workshop on Breast Imaging (IWBI 2018), 107181P (6 July 2018); doi: 10.1117/12.2316498

GDIE Event: The Fourteenth International Workshop on Breast Imaging, 2018, Atlanta, Georgia, United States 


\title{
Towards clinic-friendly solutions for patient trials in breast cancer phase contrast imaging
}

\author{
Sarah J. Lewis*a, Timur E. Gureyev ${ }^{\mathrm{b}, \mathrm{c}}$, Patrycja Baran ${ }^{\mathrm{b}}$, Seyedamir Tavakoli Taba ${ }^{\mathrm{a}}$, Serena Pacile ${ }^{\mathrm{d}, \mathrm{e}}$, \\ Christian Dullin ${ }^{\mathrm{d}, \mathrm{f}, \mathrm{g}}$, Giuliana Tromba ${ }^{\mathrm{d}}$, Daniel Hausermann ${ }^{\mathrm{h}}$, Andrew Peele ${ }^{\mathrm{h}, \mathrm{i}, \mathrm{j}}$, Darren Lockie ${ }^{\mathrm{k}}$, \\ Patrick C. Brennan ${ }^{\mathrm{a}}$ \\ ${ }^{a}$ Medical Image Optimisation and Perception Group, The University of Sydney, City Road, \\ Camperdown, NSW, Australia. ${ }^{\mathrm{b}}$ ARC Centre of Excellence in Advanced Molecular Imaging, The \\ University of Melbourne, Parkville, Victoria, Australia. ${ }^{\mathrm{c}}$ School of Science and Technology, \\ University of New England, Armidale, NSW, Australia. ${ }^{d}$ Elettra - Sincrotrone Trieste, Basovizza \\ (Trieste), Italy. ${ }^{e}$ Department of Engineering and Architecture, University of Trieste (Trieste), Italy. \\ ${ }^{\mathrm{f}}$ Department of Diagnostic and Interventional Radiology, University Hospital Goettingen, \\ Goettingen, Germany. ${ }^{\mathrm{g}}$ Max-Plank-Institute for Experimental Medicine, Göttingen, Germany. \\ ${ }^{\mathrm{h}}$ Australian Synchrotron, Australian Nuclear Science and Technology Organisation, Clayton, \\ Victoria, Australia. ${ }^{\mathrm{i}}$ Department of Chemistry and Physics, La Trobe Institute for Molecular \\ Science, La Trove University, Victoria, Australia. ${ }^{\mathrm{j} A R C}$ Centre of Excellence for Advanced \\ Molecular Imaging, Australian Synchrotron, Victoria, Australia. ${ }^{k}$ BreastScreen Victoria, VIC,
} Australia

\begin{abstract}
Phase-contrast imaging of the breast is expected to deliver significantly improved image quality and diagnostic value at a reduced radiation dose compared to present-day 2D X-ray mammography, digital breast tomosynthesis (DBT) and computed tomography (CT) and become a viable method for early diagnosis of breast cancer in women. This paper builds upon the evaluation of a novel protocol to evaluate 3D mammographic phase contrast imaging for the detection of breast cancer undertaken with a purpose designed phantom and selected breast cancer specimens. Following evaluation, propagation-based phase contrast imaging was demonstrated to have high contrast to noise ratio alongside an important reduction in radiation dose. The challenge now is to shift the focus of research to real clinic solutions, with the worldfirst demonstration of X-ray in-line full field phase-contrast mammographic tomography (PCT) with cancer patients through an international collaboration of a multi-disciplinary team.
\end{abstract}

Keywords: Breast CT, phase-contrast, synchrotron radiation, patients, cancer imaging

\section{INTRODUCTION}

\subsection{Background}

Breast cancer is the most common cancer affecting women, and over 800,000 Australians undergo mammograms each year ${ }^{1}$. Error rates in the detection of breast cancers however have remained consistently high over the last thirty years. Despite the introduction of newer technologies such as digital mammography and digital breast tomosynthesis, approximately $30 \%$ of cancers are still missed by radiologists. For patients with increased breast density, or radiologists with low experience, the missed cancer rate can be over $50 \%^{2}$. A combination of concern over radiation dose imparted to the highly radiosensitive breast tissue and the discomfort associated with mammography and digital breast tomosynthesis mainly due to tight compression and equipment design may be at least in part responsible for the low attendance rates $(55 \%)$ at screening programs encountered amongst women in Australia. Of particular note, indigenous women and highrisk women such as those with very high mammographic breast density have much lower participation rates of below $35 \%{ }^{3}$.

14th International Workshop on Breast Imaging (IWBI 2018), edited by 
In-line phase-contrast X-ray imaging was initially proposed in 1990s and implemented for the first clinical trial of planar mammography at the Elettra Synchrotron source (Trieste, Italy) at the beginnings of $2000 \mathrm{~s}^{4-5}$. However, due to the multiple technological difficulties involved, the practical implementation of this approach for medical imaging purposes has been relatively slow in its progress. With recent theoretical and technological developments, translation of this new imaging method into clinical practice is becoming feasible and in recent years, irradiation of human specimen of excised breast cancers has greatly assisted in developing optimal strategies in terms of the PCT technique and image processing. $\mathrm{X}$-ray mammography, in particular, has been recognised as the area of medical radiography which stands to gain the most from application of phase-contrast because X-ray phase-contrast provides the maximum advantage over conventional absorption-based imaging when applied to high-resolution imaging of soft tissues (such as that within the breast $)^{5}$. Recent publications have studied the performance of different variants of phase-contrast imaging of breast tissues both theoretically and experimentally on the basis of feasibility studies carried out at Elettra and at the Australian Synchrotron $^{7-11}$. At Elettra, a program aiming to implement a PCT trial using a new, single photon counting detector is ongoing, as a continuation of the first protocol of in-line phase-contrast planar mammography carried out at the beginning of the 2000s. The aim and challenge with PCT is to change breast cancer imaging in screening and symptomatic facilities internationally through a Australian-Italian collaboration to reach levels of sensitivity and specificity that exceed $95 \%$ regardless of age or ethnicity of the woman.

A PCT patient trial will begin at the Imaging and Medical beamline (IMBL) of the Australian Synchrotron in the near future. The IMBL beamline uses a super-conducting magnetic device to provide a monochromatic, nearly parallel, Xray beam with an area up to $50 \mathrm{~cm}$ (horizontal) $\times 4 \mathrm{~cm}$ at $140 \mathrm{~m}$ from the synchrotron. We will be using the energy range between 28 and $40 \mathrm{keV}$ in this study and an energy resolution of $\Delta \mathrm{E} / \mathrm{E}=10^{\wedge}-3$. Selection of an optimal detector will occur after trials with phantoms and excised tissue specimens. It is expected that the optimal detector will have pixels of approximately 50 micron size, a $14 \times 14 \mathrm{~cm}$ or larger field of view, a speed of 300-400 frames per second, 12-bit or higher dynamic range, very low electronic noise and close to $100 \%$ photon detection efficiency in the $30-40 \mathrm{keV}$ range. Some considerations for choosing these parameters are discussed below

\section{PROPOSED METHOD}

\subsection{Potential Sample (in an Australian context)}

The PCT patient trial will involve recruitment of 60 women using the full-size 3D mammographic in-line phase-contrast CT achievable at the Australian Synchrotron beamline. The aim is to have a split sample of 30 women with breast cancer and 30 women who are currently cancer-free, all who are in the 50-74 age range recommended for screening by BreastScreen Australia. All women will have previously undergone mammography and/or digital breast tomosynthesis (DBT) to either confirm cancer status or normal status. The inclusion criteria (discussed below in equipment development) is related to breast size as determined from the original mammographic imaging and the ability to remain still and comfortable for set up and exposure times longer than current technologies.

Due to the location of the IMBL in Melbourne, Australia, there is a need to recruit participants from a reasonable locale close to the research facility to reduce inconvenience. Careful consultation will be required with BreastScreen Victoria to source both cancer-free and cancer participants. Work with cancer information and consumer advocacy groups such as the Breast Cancer Network Australia (BCNA) to promote the research trial will also be necessary to ensure correct information is disseminated to the general interested population.

\subsection{Equipment development}

The PCT patient trial will require specific hardware to allow participants to remain still, comfortable and have a degree of modesty. Early modelling at the Australian Synchrotron has focused on the construction of a patient support device that will need to be developed to facilitate complete examination of all the breast tissue. PCT requires patients to be positioned prone with the exposed breast allowed to fall uncompressed through a table aperture and the head turned to the side. Due to the beam size, it is unlikely that both breasts can be examined at the same time. The PCT patient board will be required to be positioned above the X-ray beam path using a purpose designed robot.

Breast immobilization will be required, which will likely be a breast holder of a plastic material sufficiently transparent for X-rays in the targeted energy range. Immobilization will be aided by mild-suction of the breast holder to prevent micro movement. The participant sample and equipment needs are entwined, as the breast holder is likely to be limited to 
$14 \mathrm{~cm}$ in the vertical plane, allowing for the elongation of the breast due to gravity. As the breast elongation will lead to larger vertical field of view, it is desirable that the proposed vertical beam expander gives us a sufficient vertical field of view to cover the largest possible breast size in a single scan. While the image size in the vertical plane is limited only by the height of the beam and the detector, and can in principle be increased by performing several scans at different vertical positions of the patient table, the difficulty with imaging breasts larger than $14 \mathrm{~cm}$ in the horizontal plane is more critical as it is related to increased radiation dose due to the increased X-ray absorption in the tissue.

Image acquisition time will be a key determination of patient suitability to volunteer for the study, with the expected exposure times ranging from between 10 seconds to 1 minute. Throughout the acquisition, the patient table will need to be rotated at least once or more over 180 or 360 degrees, with the best-case scenario being one 180 horizontal rotation if the vertical beam expander to be installed at the IMBL can give sufficient vertical field of view to cover the whole breast.

Within the proposed scan time, the detector used will need to be fast enough to acquire the required number of frames. The required detector speed can be estimated as follows. If the horizontal breast size is, say, $12 \mathrm{~cm}$, then, at the resolution of 50 microns, this corresponds to 2400 pixels in each detector row. This, in turn, requires approximately 3600 projections for optimum reconstruction (optimum sampling conditions for uniform spatial resolution in the reconstructed CT images). In order to acquire that many projections in 10 seconds, the detector should be capable of taking 360 full frames per second, which is challenging but is within the current technology limits. Note that unlike the current situation with laboratory microfocus sources (which are not yet bright enough for this technology), the X-ray flux is not a limiting factor at synchrotron beamlines such as IMBL. The number of projections can optionally be reduced by a factor of up to 5 , if advanced CT reconstruction methods are proven to be effective in the breast PCT setting ${ }^{12}$. Figure 1 below gives a simulation of the participant positioning required.

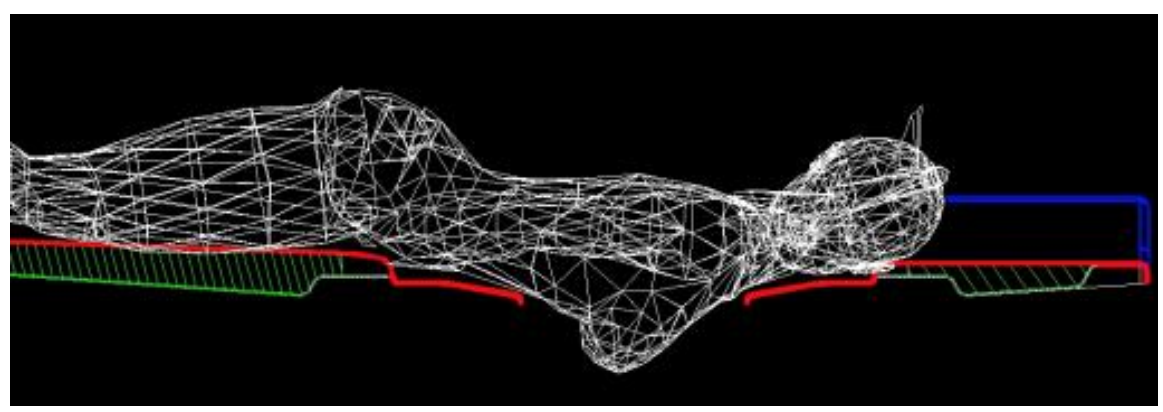

Figure 1. Schematic diagram of participant positioning required for Patient PCT trial at the IMBL Australia. Image is courtesy of the SYRMEP Triste, Italy.

\subsection{Ethical considerations}

There will be specific ethical considerations applied to PCT patient trials beginning with radiation dose. Participants will be informed that PCT radiation dose is in addition to their required care, as all recruited patients will have already undergone standard screening or diagnostic imaging procedures. In recognizing that the PCT imaging will be done in addition to, rather than instead of examinations such as standard mammography and/or tomosynthesis initially, the radiation dose applied to the women needs to be comparable or preferably lower than standard DM. Participants will be informed that the PCT technique may reveal abnormalities not visible in standard mammography or digital breast tomosynthesis, in which case they will be referred to further examination via a local service. Unfortunately, the possibility of false positive findings cannot be completely eliminated and the Participant Information Statement will explicitly make this clear.

\subsection{Reading Procedure}

The acquired images will be evaluated by a minimum of 10 radiologists using a receiver operating characteristic (ROC) technique. ROC area under the curve, sensitivity and specificity will be compared between the phase-contrast technique 
and each of mammography and DBT using standard statistical methodologies: a Student's T or Mann-Whitney U test if the data are normally or not normally distributed respectively. The radiologists used for this assessment will be recruited from Australia, Harvard Medical School and MD Anderson Cancer Centre and the online international image assessment platform Breast REader Asessment STrategy (BREAST) ${ }^{13}$ will be used as the tool for case reporting.

\subsection{Measure of Participant Satisfaction}

Participant comfort will be assessed using an exit questionnaire to draw out experiences about comfort options or individual reactions to the breast imaging experience at the IMBL. A validated five-point Likert scale for reported discomfort ${ }^{14}$ and used previously in determining patients' perspective of discomfort in mammography participants' reactions to positioning, examination duration, immobilization, interaction with equipment and emotional discomfort will be employed ${ }^{15}$. For conventional mammography and DBT, participants will be asked to select levels of discomfort from none, slight, moderate, considerable or severe. After clinically trialing the PCT patient support table, the same scale will be used to investigate patients' perspectives with PCT. An opportunity to provide open ended responses will be available to patients regarding their experiences of being part of an experimental technique.

\section{RESULTS}

\subsection{Early results with specimens}

Image analysis of recent work by the authorship team using an in-line phase-contrast approach was conducted using the variables of distance between the sample and the detector, X-ray energy, reconstruction method and the use of phase retrieval in image processing. Using expert observers, our work found that long propagation sample-to-detector distance, sufficiently low X-ray energy beam and correct level of application of phase retrieval algorithm in conjunction with CT reconstruction methods resulted in a substantial improvement in radiological quality of images. Indeed, the best radiological scores were attributed to the following imaging condition: $9.81 \mathrm{~m}$ distance and $32 \mathrm{keV} \mathrm{X}$-ray energy at approximately $4 \mathrm{mGy}$ dose. Several combinations of reconstruction methods and phase retrieval application could result in high quality images ${ }^{8}$. See Figure 2 for one example of such high quality PCT mammography images.

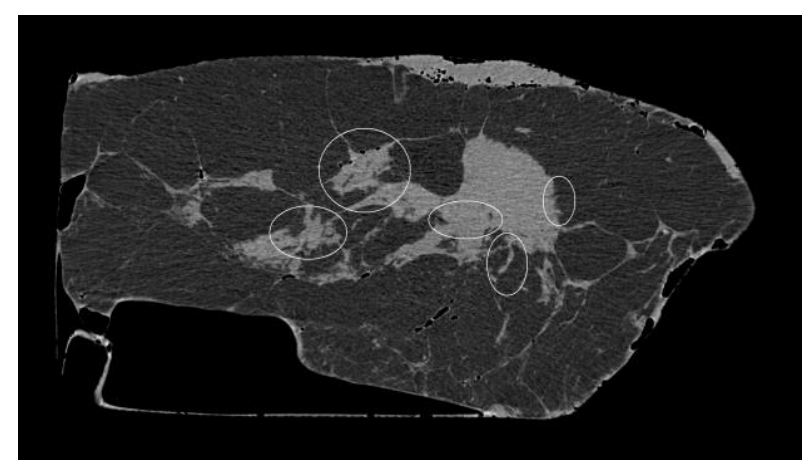

Figure 2. Mastectomy sample imaged at the SYRMEP beamline of Elettra using PCT method under conditions reported above 


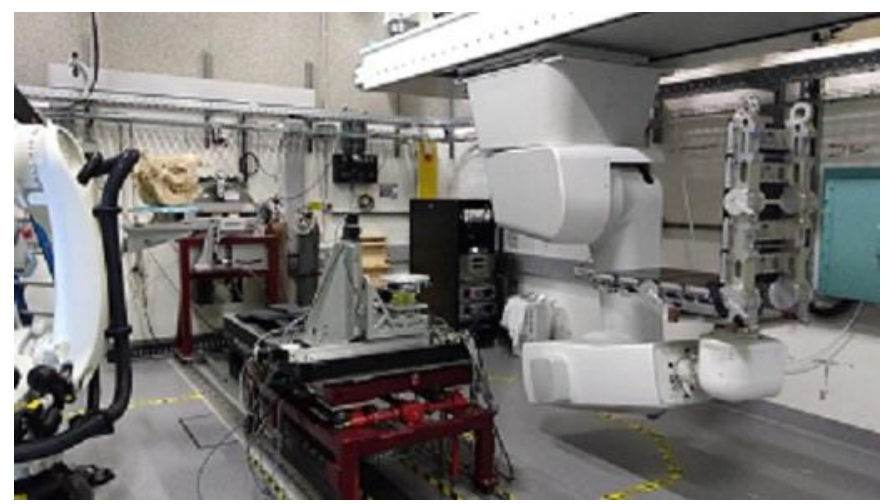

Figure 3. Hutch 3B of the IMBL at the Australian Synchrotron. Image shows on the left: the robot used to scan large animals, to the right of the rhino skull: the entrance port of the X-rays, in the centre on the table: the small CT stage used for mastectomy samples, on the right: the patient positioning robot which chair will be replaced by the patient support table for this work

\section{DISCUSSION}

Although the 6-9 m long object to detector distance as reported above would not generally be considered feasible in a clinic situation, it can be implemented at specialized synchrotron beamlines such as IMBL at the Australian Synchrotron. This distance can be potentially reduced to approximately $2 \mathrm{~m}$ in a future clinical implementation. The required object to detector distance is paramount to the requirements of a clinic set-up, including the important need to remain connected to the patient/clients via line of sight, audio contact and with a room that allows women to feel confident with an experimental technique. While most participants would be familiar with the idea of X-ray imaging through general population knowledge of BreastScreen, as well as their own personal involvement, an introductory video may be helpful in explaining the PCT technique in lay terms as well offering simple explanations about why compression is unnecessary.

Studies have also shown that cancer patients need specific education about the value of being involved in research trials ${ }^{16}$ and recently diagnosed patients experience spikes in stress and adjustment reactions requiring psychosocial care ${ }^{17}$. Equally, although there is little published about strategies for recruitment of women to trial medical imaging as linked to cancer diagnosis, some authors through commentary articles have reported that cancer patients are more likely to participate in pharmaceutical research if there is a longer period of time between initial diagnosis and the beginning of treatment/surgery ${ }^{18}$ and that participation by cancer patients can be enhanced by the immediate referral by a doctor, such as a radiologist or surgeon ${ }^{19}$.

The proposed PCT Patient Trial needs careful planning to ensure the diagnostic efficiency of PCT can be measured. Specifically, recruitment of a valid sample is a real challenge that requires stakeholder involvement to ensure that women are supported through the experimental imaging process. Recent advances in detector efficiency and image acquisition modelling have allowed object to detector distances to be reduced to a more clinic friendly scenario. The latest findings and conclusions that will impact upon the patient trial will be presented with specific focus on imaging protocols, survey development and preliminary results.

\section{REFERENCES}

[1] Cancer Australia., "Breast cancer statistics: Breast cancer in Australia," https://breastcancer.canceraustralia.gov.au/statistics (13th December 2017).

[2] Lauby-Secretan, B., et al., "Breast-cancer screening-viewpoint of the IARC Working Group," New England Journal of Medicine, 372(24), 2353-8 (2015). 
[3] Australian Institute of Health and Welfare., [BreastScreen Australia monitoring report 2011-2012 Cancer Series no. 86. Cat. no. CAN 83]. AIHW, Canberra (2014).

[4] Wilkins, S., Gureyev, T., Gao, D., Pogany, A. \& Stevenson, A., "Phase-contrast imaging using polychromatic hard X-rays," Nature 384, 335-338 (1996)

[5] Castelli, E., et al., "Mammography with Synchrotron Radiation: First Clinical Experience with Phase-Detection Technique," Radiology 259, 684-694 (2011).

[6] Raupach, R. \& Flohr, T., "Performance evaluation of x-ray differential phase contrast computed tomography (PCT) with respect to medical imaging," Medical Physics 39, 4761-4774 (2012).

[7] Pacile, S., et al., "Clinical application of low-dose phase contrast breast CT: methods for the optimization of the reconstruction workflow," Biomed Opt Express 6, 3099-3112 (2015).

[8] Baran, P., et al., "Optimization of in-line X-ray phase-contrast tomographic imaging for breast cancer screening, "Phys.Med.Biol 62(6), 2315-2332 (2017).

[9] Longo, R., et al., "Towards breast tomography with synchrotron radiation at Elettra: first images," Physics in Medicine and Biology 61, 1634 (2016).

[10] Tromba, G., et al., "Phase-Contrast Clinical Breast CT: Optimization of Imaging Setups and Reconstruction Workflows," Breast Imaging $13^{\text {th }}$ International Workshop IWDM, 625-35 (2015).

[11] Nesterets, Y. I. et al., "A feasibility study of X-ray phase-contrast mammographic tomography at the Imaging and Medical beamline of the Australian Synchrotron," J Synchrotron Radiat 22, 1509-1523 (2015).

[12]Zhao, Y., et al., "High-resolution, low-dose phase contrast X-ray tomography for 3D diagnosis of human breast cancers," PNAS 109(45), 18290-18294 (2012).

[13] Tapia, K., Lee, W., and Brennan, P., "Breast Screen Reader Assessment Strategy: Transforming Breast Cancer Diagnosis Globally: A Mini Review," Int J Radiol Radiat Ther 4(1) (2017).

[14] Frank, A. J. M., Moll, J. M. H. and Hort, J. F., "A comparison of three ways of measuring pain," Rheumatology 21, 211-217, (1982).

[15] Poulos, A., McLean, D., Rickard, M.and Heard, R., "Breast compression in mammography: how much is enough?" Australasian Radiology 47, 121-126 (2003).

[16] New South Wales Cancer Institute., "Taking part in a clinical trial" https://www.cancerinstitute.org.au/dataresearch/clinical-trials/taking-part-in-a-clinical-trial (4th January 2018)

[17] Beatty, L., Oxlad, M., Koczwara, B., and Wade, TD., "The psychosocial concerns and needs of women recently diagnosed with breast cancer: a qualitative study of patient, nurse and volunteer perspectives," Health Expect 11(4), 331-42 (2008).

[18] Pinkowish, M., "Recruitment to breast cancer clinical trials: what makes a difference," Cancer J. Clin 59(1) 1-2 (2009).

[19] Sygna, K., Johanson, S., and Ruland, C., "Recruitment challenges in clinical research including cancer patients and their caregivers. A randomized controlled trial study and lessons learned," Trials 16 (428) (2015). 